J3eA - Vol. 2 - 9 (2003).

DOI : 10.1051/bib-j3ea:2003009

\title{
FFT et applications : caractérisation d'un CAN, choix d'une fenêtre de pondération et réponse en fréquence d'un système linéaire
}

\author{
G. Couturier ${ }^{*}{ }^{*}$, L. Béchou ${ }^{* *}$, J.B. Bégueret ${ }^{* * *}$ et C. Pellet ${ }^{* * * *}$ (IUT Université Bordeaux I) \\ Mis en ligne le 27 juin 2003.
}

\begin{abstract}
Résumé
Les transformées temps-fréquence (transformée de Fourier, transformée en ondelettes) sont aujourd'hui couramment utilisées en traitement du signal. Dans cet article on se focalise uniquement sur la transformée de Fourier et plus particulièrement sur la transformée de Fourier discrète rapide (FFT pour Fast Fourier Transform). L'interprétation d'une FFT reste cependant délicate car il s'agit avant tout du résultat d'un algorithme de calcul et une bonne dose de mathématiques est a priori nécessaire pour comprendre les subtilités de la FFT. Avec le développement considérable de l'informatique et de l'instrumentation, la FFT n'est plus la seule propriété des chercheurs et ingénieurs et l'outil FFT s'est largement « démocratisé » : pratiquement tous les oscilloscopes numériques sont aujourd'hui pourvus d'un menu FFT. En conséquence, de nombreux techniciens, maîtrisant peu ou mal les mathématiques, sont confrontés à l'interprétation des FFT. Plutôt que de longs développements mathématiques, nous proposons aux étudiants de niveau bac +2 de découvrir l'outil FFT et ses applications aux travers de diverses expériences décrites dans cet article.
\end{abstract}

Mots-clés : FFT, fenêtre de pondération, conversion analogique-numérique, bruit de quantification, réponse en fréquence d'un système linéaire.

(C) EDP Sciences, 2003.

Niveau des étudiants. L'article s'adresse principalement à des étudiants possédant un niveau de première année IUT, BTS et DEUG.

* Gérard Couturier ${ }^{1}$, ancien élève de l'École Nationale Supérieure d'Électronique, Informatique et

Radiocommunications de Bordeaux (ENSEIRB), est actuellement Professeur des Universités à l'IUT de Bordeaux

I. Son activité de recherche se déroule au Centre de Physique Moléculaire Optique et Hertzienne (CPMOH) dans le groupe « Nanophysique par sonde locale de force », et concerne d'une manière générale l'étude des propriétés des matériaux à l'échelle du nanomètre.

o e-mail : gerard.couturier@iut.u-bordeaux1.fr (auteur de correspondance)

** L Béchou ${ }^{2}$, est actuellement Maître de Conférences à l'IUT Bordeaux I au département Génie Electrique et Informatique Industrielle. Il enseigne l'Électronique analogique et numérique ainsi que la Physique en première et deuxième année. Il est rattaché au Laboratoire de microélectronique IXL de l'université de Bordeaux I où il effectue des recherches dans le domaine de la fiabilité des composants et systèmes optoélectroniques pour des applications de télécommunication.

e-mail : bechou@ixl.u-bordeaux1.fr

*** Jean-Baptiste Bégueret ${ }^{2}$, a reçu le DESS de Microélectronique en 1990 et la thèse de doctorat en électronique en 1996, à l'Université de Bordeaux I. Depuis 1997, il est Maître de Conférences à l'IUT GEII de Bordeaux où il enseigne l'électronique analogique en seconde année. Ses recherches s'effectuent au Laboratoire de Microélectronique IXL de l'Université de Bordeaux I, dans le cadre de la conception de circuits intégrés radiofréquences. 
**** Claude Pellet ${ }^{2}$, ancien élève de l'ENS de Cachan, occupe depuis 1993 un poste de Professeur des Universités à l'IUT de Bordeaux I. Son activité de recherche se déroule au Laboratoire IXL au sein de l'opération « capteurs microsystèmes » et concerne l'évaluation d'assemblage, la fiabilité des microsystèmes et la conception de microsystèmes pour des applications médicales.

e-mail : pellet@ixl.u-bordeaux1.fr

\footnotetext{
${ }^{1}$ CPMOH, UMR CNRS 5798, Université Bordeaux I, 351 cours de la Libération, F-33405 Talence Cedex, France.

${ }^{2}$ Laboratoire IXL, UMR CNRS 5818, Université Bordeaux I, 351 cours de la Libération, F-33405 Talence Cedex, France.
}

\section{Introduction}

L'algorithme de FFT popularisé par Cooley et Tuckey dès 1965 et l'accès aisé aux moyens de calculs font aujourd'hui de la transformée de Fourier discrète un outil incontournable pour tous les étudiants suivant un cursus EEA (Électronique Électrotechnique et Automatique). L'article concerne plus particulièrement l'apprentissage de la FFT au niveau bac+2 dans les départements GEII des IUT. C'est ainsi que les modules MA2 (Mathématiques du signal numérique), EN22 (Traitement du signal numérique) et AU21 (Automatique : modélisation et analyse des systèmes linéaires) du Programme Pédagogique National [1] des départements GEII sont directement concernés par la transformée de Fourier et l'outil FFT. D'un point de vue chronologique, la transformée de Fourier est abordée en Mathématiques, après l'introduction des séries de Fourier. Les transformées de Fourier numérique et discrète ainsi que la FFT sont ensuite traitées principalement dans les enseignements d'électronique. Pourquoi ? Parce qu'il est plus facile d'aborder cet outil après avoir présenté l'échantillonnage et la conversion analogiquenumérique. En effet, dans notre cas les signaux traités sont issus d'une chaîne d'acquisition, nous ne pouvons donc pas faire l'économie du bruit de quantification. Grosso modo, une heure de cours est consacrée à la FFT, les problèmes soulevés par l'outil (nombre de points finis, choix de la fenêtre...) sont abordés en utilisant des exemples concrets de FFT obtenues au moyen d'un oscilloscope numérique et de générateurs de signaux. Un TD d'environ deux heures est consacré à une étude de cas : le relevé de la réponse en fréquence d'un système linéaire et à l'exploitation de quelques FFT. Un TP de trois heures permet aux étudiants de réaliser les expériences décrites au $\S 3$ de cet article :

- $\quad$ choix d'une fenêtre de pondération ;

- mesure du bruit de quantification d'un convertisseur analogique-numérique (CAN) et des non-linéarités de la caractéristique de transfert;

- étude de la réponse en fréquence d'un système linéaire par le calcul de la transformée de Fourier de la réponse impulsionnelle.

Le menu FFT des oscilloscopes numériques est également utilisé au cours de divers TP pour, par exemple, visualiser des spectres ou encore mesurer des taux de distorsion.

La mesure du bruit de quantification n'est pas sans poser quelques difficultés liées : i) au problème de cohérence entre signal et bruit et, $\mathrm{ii}$ ) au rôle du bruit physique dans la mesure. La discussion concernant ces deux points est reportée au $\S 4$ car elle n'est pas indispensable dans une première approche et ne peut être raisonnablement incluse dans une présentation à bac +2 . Le $\S 2$ de l'article présente très brièvement les bases de la FFT.

\section{Préliminaires}

La transformée de Fourier $X(f)$ d'un signal $x(t)$ continu dans le temps s'écrit [2] :

$$
X(f)=\int_{-\infty}^{\infty} x(t) e^{-j 2 \pi f t} d t
$$


Après échantillonnage de $x(t)$, on obtient les échantillons $x\left(k T_{e}\right)$ où $T_{e}$ désigne la période d'échantillonnage du signal $x(t)$. La transformée de Fourier d'un signal échantillonné est alors périodique de période $F_{e}=1 / T_{e}$ et son module est une fonction paire de la variable $f$. La transformée de Fourier numérique $X_{\text {num }}(f)$ des échantillons $x\left(k T_{e}\right)$ s'écrit alors [3-5]:

$$
X_{n u m}(f)=\sum_{k=-\infty}^{\infty} x\left(k T_{n}\right) \mathrm{e}^{-j 2 \pi f k T_{*}}
$$

Un calculateur ne permet pas d'évaluer la transformée de Fourier $X_{\text {num }}(f)$ pour l'ensemble infini des valeurs de $k$. En pratique, le nombre d'échantillons est limité à $M$ points par la multiplication des échantillons $x\left(k T_{e}\right)$ par les $M$ échantillons $y\left(k T_{e}\right)$ d'une fenêtre de pondération encore appelée fenêtre d'analyse ou d'apodisation. Pour simplifier l'écriture, les échantillons $x\left(k T_{e}\right)$ et $y\left(k T_{e}\right)$ sont notés par la suite $x(k)$ et $y(k)$. Les échantillons $y(k)$ de la fenêtre sont nuls pour $k<0$ et $k>M-1$, soient $z(k)=x(k) y(k)$ les échantillons issus de la multiplication des $x(k)$ par les $y(k)$ pour $k \in[0,1, \cdots, M-1]$

. La transformée de Fourier numérique $Z_{\text {num }}(f)$ des $M$ échantillons $z(k)$ s'écrit alors :

$$
Z_{\text {num }}(f)=\sum_{k=-\infty}^{\infty} z(h) \mathrm{e}^{-j I \pi f k T .}
$$

Avec un calculateur, il est impossible de calculer $Z_{\text {num }}(f)$ pour toutes les valeurs de ${ }^{f \in\left[0, F_{e}\right]}$. Comme $Z_{\text {num }}(f)$ est périodique de période $F_{e}$ dans l'espace des fréquences, on ne calcule $Z_{\text {num }}(f)$ que pour les valeurs de $f$ telles que $f=n \frac{F_{\mathrm{e}}}{M} \quad n \in[0,1, \ldots, M-1]$ discrète : , on obtient ainsi les composantes $Z(n)$ de la transformée de Fourier

$$
Z(n)=Z_{\text {num }}\left(f=n \frac{F_{e}}{M}\right)=\sum_{k=0}^{M-1} z(k) e^{-j z \pi n k / M}
$$

Dans le cas où le nombre de points d'acquisition $M$ est une puissance de $2\left(M=2^{p}\right)$, on dispose alors d'algorithmes de calculs rapides, le plus connu d'entre eux est l'algorithme à entrelacement temporel de Colley et Tuckey. Cet algorithme de FFT permet de ramener le nombre de multiplications à $(M p / 2)$. Si $M$ est différent d'une puissance de 2, on utilise un algorithme dit de DFT (Direct Fourier Transform) et le nombre de multiplications à effectuer est égal à $M^{2}$. On peut toujours ramener le nombre de points $M$ à une puissance de 2 , soit en éliminant des points, soit encore en ajoutant des zéros, c'est la technique de remplissage ou bourrage ou encore "zero padding" en anglais. Ajouter par exemple $Q$ zéros ne modifie pas la transformée de Fourier numérique $Z_{\text {nиm }}(f)$, par contre les composantes $Z(n)$ de la transformée de Fourier discrète sont calculées non plus pour $f=n F_{e} / M$ mais pour $f=n F_{e} /(M+Q)$ où $(M+Q)$ est maintenant une puissance de 2.

\section{Expériences}

\subsection{Choix d'une fenêtre de pondération}

Les échantillons $z(k)$ sont donc égaux au produit des $x(k)$ par les échantillons $y(k)$ de la fenêtre. À une multiplication dans le domaine du temps correspond une convolution circulaire dans le domaine des fréquences : $Z_{\text {mum }}(f)=X_{\text {тum }}(f) \otimes Y_{\text {rum }}(f)$ différentes des valeurs de $X_{n u m}\left(f=n F_{e} / M\right)$, elles-mêmes différentes des valeurs de $X\left(f=n F_{e} / M\right)$. La fenêtre idéale serait celle dont la transformée de Fourier numérique $Y_{\text {num }}(f)$ serait équivalente à un Dirac périodique, soit encore un peigne de Dirac, malheureusement ceci correspond à un nombre de points $M$ infini. 
En conséquence, une fenêtre de pondération altère l'allure des spectres. L'expérimentateur dispose de diverses fenêtres se caractérisant dans le domaine temporel par les valeurs des $y(k)$, constantes et égales à 1 par exemple pour une fenêtre rectangulaire, de type cosinus pour une fenêtre de Hanning, etc. La transformée de Fourier numérique $Y_{\text {num }}(f)$ des $y(k)$ joue un rôle fondamental en analyse spectrale. Une fenêtre de pondération est caractérisée par deux paramètres principaux :

- la largeur du lobe principal de $Y_{\text {num }}(f)$ définit la résolution de l'analyse, c'est-à-dire l'aptitude à distinguer deux fréquences proches l'une de l'autre ;

- la décroissance des lobes secondaires qui définit la dynamique de l'analyse, c'est-à-dire l'aptitude à faire apparaître deux fréquences éloignées l'une de l'autre et d'amplitudes très différentes.

Sachant que la fenêtre idéale est un Dirac, ces deux paramètres sont deux indicateurs qui renseignent grosso modo de combien la fenêtre utilisée s'éloigne de la fenêtre idéale. Le choix d'une fenêtre de pondération [6] est un problème non trivial surtout si on dispose de peu d'information sur le signal. D'un point de vue pédagogique, il est par contre relativement aisé d'expliquer le choix d'une fenêtre ; il suffit de choisir des situations où les concepts de résolution et dynamique sont faciles à mettre en oeuvre. Pour cette première expérience nous utilisons deux synthétiseurs de signaux numériques arbitraires HP33120A [7], un additionneur à 3 résistances et un oscilloscope numérique Yokogawa DL1400 (1 Gb/s, $150 \mathrm{MHz}, 8$ bits) pour l'enregistrement [8].

Les FFT sont disponibles soit directement sur l'oscilloscope numérique, soit encore par calcul en utilisant le logiciel Matlab. Dans ce dernier cas, les données stockées dans la mémoire de l'oscilloscope (profondeur mémoire 10000 points au maximum) sont transférées vers le calculateur via le bus GPIB. L'oscilloscope numérique Yokogawa DL1400 ne dispose que des fenêtres rectangulaire et de Hanning, du point de vue pédagogique c'est amplement suffisant pour illustrer les notions de résolution et dynamique.

En préambule au choix de la fenêtre, on propose l'expérience simple suivante : le signal à analyser est de type sinusoïdal, de fréquence $F_{I N}$ et une fenêtre rectangulaire est utilisée. En faisant varier la fréquence $F_{I N}$, on constate que les fuites spectrales s'annulent si $F_{I N}=K F_{e} / M$ où $M$ est le nombre de points d'acquisition, $F_{e}$ la fréquence d'échantillonnage et $K$ un entier. Dans le cas où $F_{I N} \neq K F_{e} / M$, on pourra faire observer que les fuites spectrales sont fortement atténuées quand on choisit la fenêtre de Hanning. Dans le cas particulier où $F_{I N}=K F_{e} / M$ les composantes $Z_{n}$ sont calculées pour des fréquences $f=n F_{e} / M$ telles que $Z_{n u m}\left(f=n F_{e} / M\right)=0$, excepté pour la fréquence particulière $f=F_{I N}=K F_{e} / M$.

La fenêtre de Hanning conduit cependant à un élargissement du lobe principal affectant la résolution en fréquence. La largeur du lobe principal de la fenêtre rectangulaire est égale à $2 F_{e} / M$ alors qu'elle est de $4 F_{e} / M$ pour une fenêtre de Hanning. La fenêtre rectangulaire ne permet pas par contre d'obtenir une bonne dynamique car les lobes secondaires décroissent très lentement. L'obtention d'une meilleure dynamique, pour un nombre de points $M$ donné, peut être obtenue par utilisation des fenêtres comme Hanning, Hamming.... Le choix de la fenêtre se fait en fonction des écarts de fréquence et d'amplitude des diverses composantes contenues dans le signal.

Pour appréhender le choix d'une fenêtre, on choisit maintenant deux situations illustrant le compromis résolutiondynamique.

1. Le signal à analyser est la somme de deux de fréquences $F_{1}$ et $F_{2}$ très proches l'une de l'autre et d'amplitudes identiques, $F_{1}=48828,125 \mathrm{~Hz}$ et $F_{2}=49072,265 \mathrm{~Hz}$. Le zoom autour des fréquences $F_{1}$ et $F_{2}$ de la FFT, calculée avec $M=8192$ et une fenêtre rectangulaire, est donné sur la figure 1, il fait clairement apparaître les deux fréquences désirées, les FFT sont normalisées à l'amplitude maximale. On remarquera que les deux fréquences $F_{1}$ et $F_{2}$ sont respectivement des multiples du pas de calcul, c'est-àdire de $F_{e} / M$ avec $F_{e}=1 \mathrm{MHz}$, en effet $F_{1}=400 F_{e} / M$ et $F_{2}=402 F_{e} / M$, c'est la raison pour laquelle les fuites spectrales sont nulles. La FFT de la figure 2, calculée avec une fenêtre de Hanning, ne permet plus de séparer les deux fréquences car la largeur du lobe principal $4 F_{e} / M$ est supérieure à l'écart de fréquence $\left(F_{2}-F_{1}\right)=2 F_{e} / M$.

2. Le signal à analyser est maintenant la somme de deux fréquences $F_{1}=48889 \mathrm{~Hz}$ et $F_{2}=61096 \mathrm{~Hz}$, éloignées l'une de l'autre et d'amplitudes très différentes, dans un rapport d'environ $10^{3}$. Le zoom autour des fréquences $F_{1}$ et $F_{2}$ de la FFT calculée avec une fenêtre rectangulaire est donné à la figure 3. La FFT ne fait pas apparaître la composante de très faible amplitude. En effet, la fréquence $F_{1}=400,5 F_{e} / M$, en conséquence les fuites spectrales dues à cette composante sont importantes à la fréquence $F_{2}$ et masquent cette dernière. La FFT de la figure 4, calculée avec une fenêtre de Hanning, fait quant à elle clairement 
apparaître les deux fréquences. La fenêtre de Hanning n'est pas en toute rigueur le meilleur choix, en effet il existe des fenêtres avec des fuites spectrales plus faibles et donc mieux adaptées pour traiter ce problème.

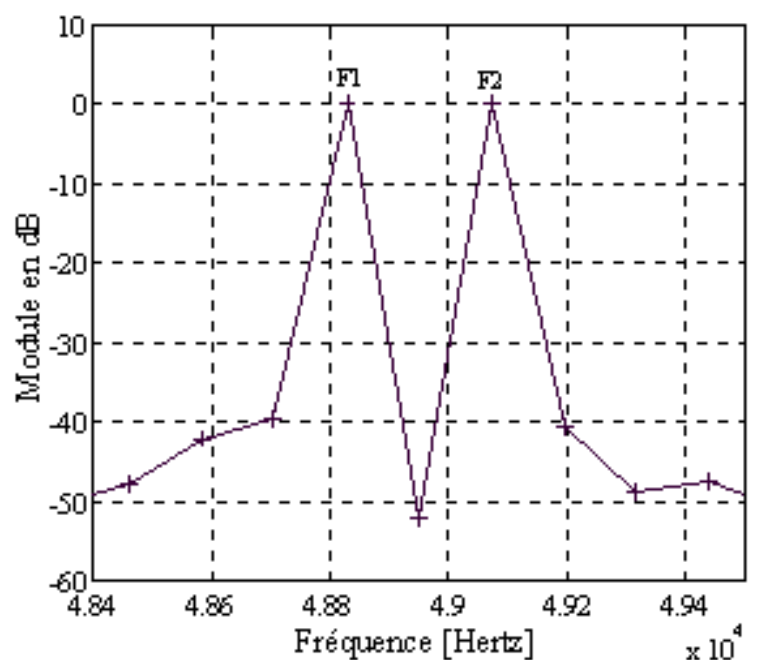

Fig. 1. Zoom autour des fréquences $F_{1}$ et $F_{2}$ de la FFT calculée avec une fenêtre rectangulaire, les deux fréquences $F_{1}$ et $F_{2}$ sont proches l'une de l'autre $\left(F_{2}\right.$ $\left.F_{1}\right)=2 F_{e} / M$; résultat expérimental.

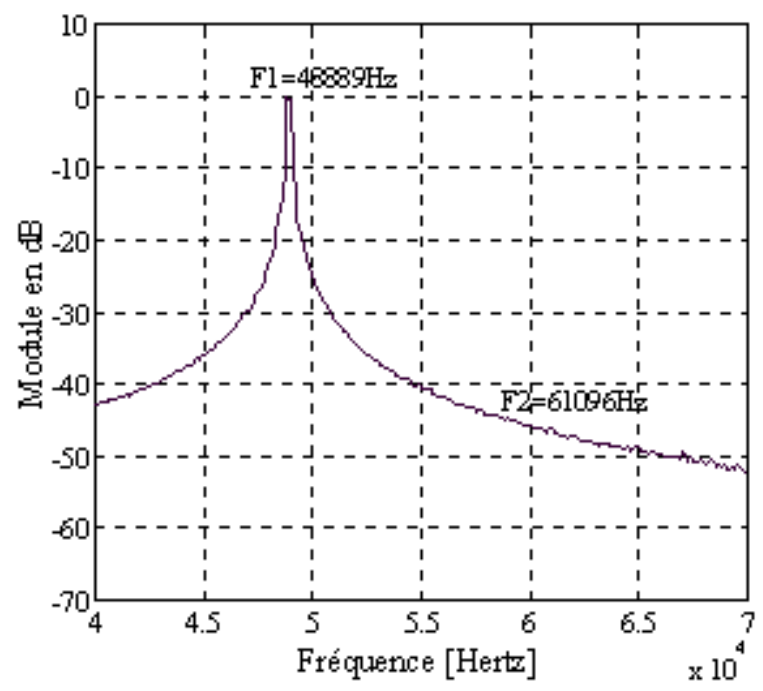

Fig. 3. Zoom autour des fréquences $F_{1}$ et $F_{2}$ de la FFT calculée avec une fenêtre rectangulaire, les deux fréquences $F_{1}$ et $F_{2}$ sont éloignées l'une de l'autre et d'amplitudes très différentes ; résultat expérimental.

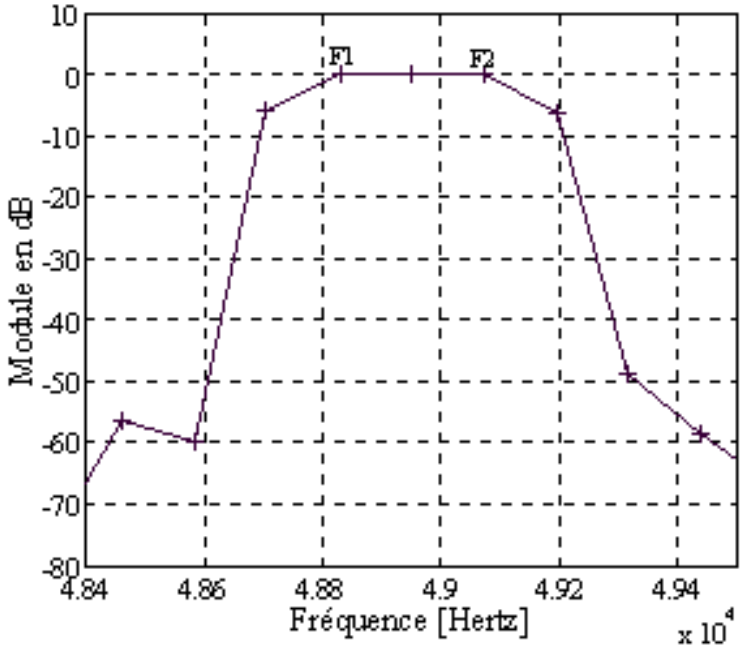

Fig. 2. Zoom autour des fréquences $F_{1}$ et $F_{2}$ de la FFT calculée avec une fenêtre de Hanning, les deux fréquences $F_{1}$ et $F_{2}$ sont proches l'une de l'autre $\left(F_{2}\right.$ $\left.F_{1}\right)=2 F_{e} / M$; résultat expérimental.

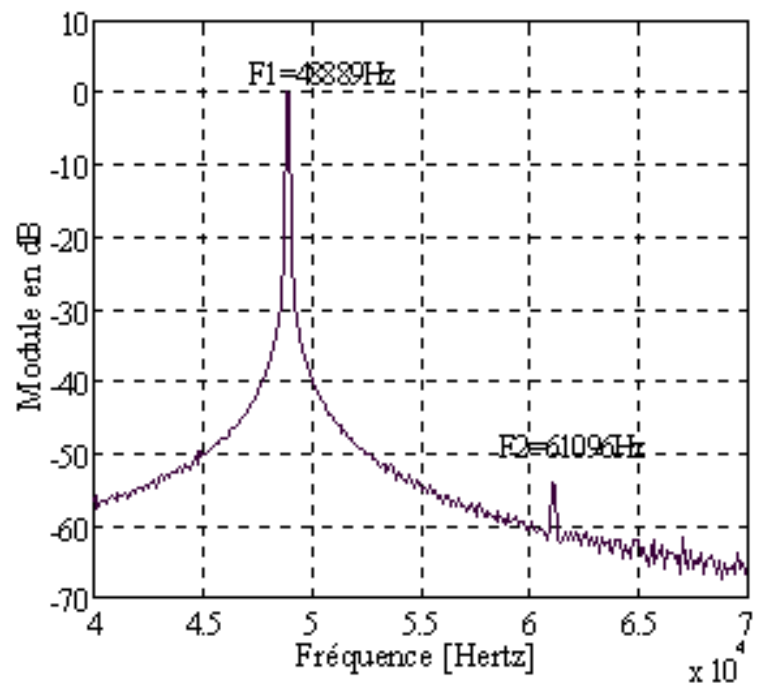

Fig. 4. Zoom autour des fréquences $F_{1}$ et $F_{2}$ de la FFT calculée avec une fenêtre de Hanning, les deux

fréquences $F_{1}$ et $F_{2}$ sont éloignées l'une de l'autre et d'amplitudes très différentes ; résultat expérimental.

\subsection{Caractérisation d'un convertisseur analogique numérique}

Un convertisseur analogique numérique (CAN) permet de convertir des échantillons analogiques en un code binaire de $N$ bits. Le choix d'un CAN (flash, SAR, sigma-delta...) se fait en fonction de la résolution souhaitée (nombre de bits $N$ ), de la distorsion acceptable et surtout du temps de conversion imposé par la période d'échantillonnage $T_{e}=1 / F_{e}$, la fréquence d'échantillonnage $F_{e}$ est quant à elle imposée par le théorème de Shannon.

L'opération de quantification réalisée par un CAN introduit un bruit appelé bruit de quantification : c'est la différence entre la valeur réelle des échantillons avant la conversion et la valeur restituée à partir du seul code de $N$ bits en prenant le milieu du quantum. Pour un CAN de $N$ bits, le rapport Signal/Bruit de quantification (SNR pour 
Signal Noise Ratio $)$ est égal à : $S N R=\left(20 \log _{10} 2^{N}\right)=(6,02 N)_{\mathrm{dB}}$ si le signal à convertir est équi-réparti dans la pleine échelle (FSR pour Full Scale Range) du CAN. La démonstration dans le cas général nécessite en toute rigueur l'utilisation de la notion de variance, on peut cependant obtenir le résultat dans le cas particulier d'un signal triangulaire en utilisant les valeurs efficaces mieux maîtrisées par l'ensemble des étudiants. Dans le cas d'un signal test sinusoïdal, l'expression du $S N R$ doit être corrigée pour tenir compte du fait que le signal n'est pas exactement équi-réparti, on obtient alors : $S N R=(6,02 N+1,76)_{\mathrm{dB}}$.

On se propose de mettre en évidence le bruit de quantification et d'estimer le $S N R$ du CAN de l'oscilloscope numérique Yokogawa en utilisant l'outil FFT. La fréquence $F_{I N}$ du signal appliqué à l'entrée de l'oscilloscope est voisine de $50 \mathrm{kHz}$, en toute rigueur $50170,898 \mathrm{~Hz}$ pour éliminer les fuites spectrales, la fréquence

d'échantillonnage $F_{e}=1 \mathrm{MHz}$ et $M=8192$, par conséquent

$$
50170,898 \mathrm{~Hz}=\frac{F_{e}}{M} K
$$

calculée en utilisant une fenêtre rectangulaire, le résultat est montré à la figure 5. En première lecture, la FFT fait apparaître le fondamental à $50 \mathrm{kHz}$ et un plancher moyen de bruit à environ - $85 \mathrm{~dB}$. L'amplitude de la sinusoïde conduisant à la FFT de la figure 5 est d'environ $1 \mathrm{~V}$, la sensibilité de l'oscilloscope est ajustée de telle manière que le signal occupe la totalité de l'écran. Dans les mêmes conditions, avec une amplitude de $10 \mathrm{mV}$, le plancher de bruit remonte et atteint environ - $70 \mathrm{~dB}$, dans ce cas le plancher de bruit est dominé par le bruit physique et le bruit de quantification ne peut être mesuré. Avec une amplitude de $1 \mathrm{~V}$, les bruits physiques sont quasiment négligeables (voir la discussion du $\S 4$ ) et dans ce cas le plancher moyen de bruit dépend du SNR et de la FFT. La valeur théorique attendue est : $6,02 N+1,76+10 \log _{10}(M / 2)$ où $M$ est le nombre de points de calcul de la FFT [9]. Sans entrer dans le détail de cette relation, rappelons que le module de la FFT, calculé avec une fenêtre rectangulaire, d'un signal sinusoïdal de fréquence $F_{I N}$, d'amplitude $A$ et échantillonné avec $M$ points est égal à $A M / 2$ si la fréquence $F_{I N}$ est un multiple de $F_{e} / M$. Dans le cas de la figure 5, le plancher théorique de bruit est donc attendu à - $86 \mathrm{~dB}$. Bien que le nombre $N$ de bits du CAN soit fixé, il est intéressant pédagogiquement de simuler un CAN avec un nombre de bits inférieur à 8. Sans modifier l'amplitude du signal d'entrée, le simple fait de multiplier par exemple l'échelle verticale (Volts/Div) par 8 permet de simuler un CAN de 5 bits. On peut aussi changer l'amplitude du signal, il faut simplement que la trace sur l'écran occupe seulement le 1/8 de l'écran total. On observe bien alors une remontée du plancher de bruit d'environ $18 \mathrm{~dB}$.

La FFT de la figure 5, fait apparaître d'autres pics, principalement à $100 \mathrm{~Hz}, 150 \mathrm{~Hz}, 200 \mathrm{~Hz}$ et $250 \mathrm{~Hz}$ respectivement. Ces pics proviennent des harmoniques du générateur et/ou des non-linéarités de l'ensemble amplificateur plus CAN de l'oscilloscope numérique. Le taux de distorsion mesuré à partir des pics de la figure 5 conduit à une valeur d'environ 4,5.10 ${ }^{-3}$, soit - $47 \mathrm{dBc}$. Le constructeur du générateur annonce une distorsion harmonique de $-60 \mathrm{dBc}$ ( $c$ pour carrier) pour des fréquences $F_{I N}$ comprises entre $20 \mathrm{kHz}$ et $100 \mathrm{kHz}$, on peut donc raisonnablement conclure que les pics sont dus en partie aux non-linéarités de l'amplificateur plus CAN de l'oscilloscope.

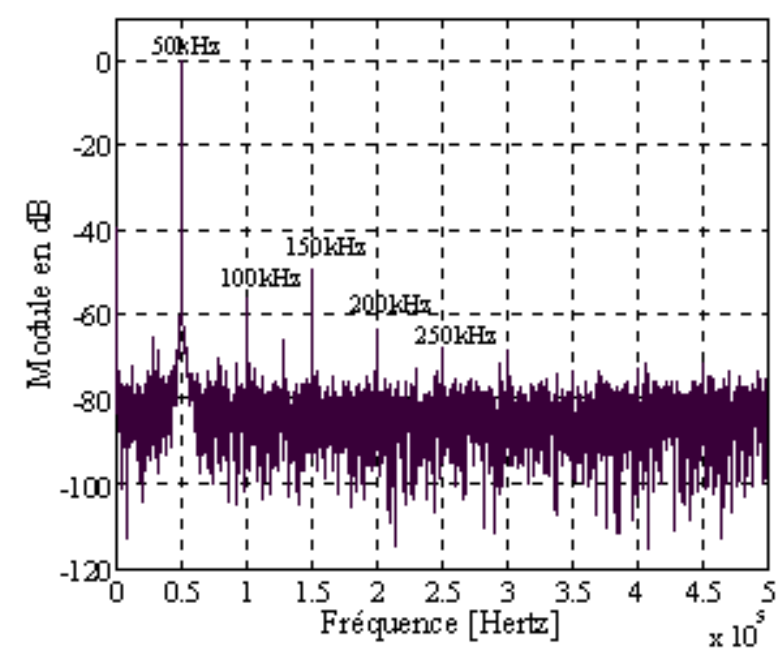

Fig. 5. FFT avec fenêtre rectangulaire d'un signal sinusoïdal de fréquence $F_{I N}=50170,898 \mathrm{~Hz}$ échantillonné à $1 \mathrm{MHz}$ et codé avec 8 bits ; résultat expérimental.

Pour mettre en évidence les non-linéarités de l'ensemble amplificateur plus CAN, on peut faire appel à la méthode couramment utilisée en électronique pour la mesure du taux d'intermodulation des amplificateurs et des 
mélangeurs. Cette technique consiste à appliquer à l'entrée de l'amplificateur plus CAN un signal constitué par la somme de deux fréquences $F_{1}$ et $F_{2}$ proches l'une de l'autre, le terme non linéaire d'ordre 3 conduit à l'apparition des fréquences $\left(2 F_{1}-F_{2}\right)$ et $\left(2 F_{2}-F_{1}\right)$. La figure 6 montre la FFT obtenue en prenant $F_{1}=250 \mathrm{kHz}$ et $F_{2}=260 \mathrm{kHz}$. Les deux raies observées à $240 \mathrm{kHz}$ et $270 \mathrm{kHz}$ sont donc la signature de la non-linéarité de l'ensemble amplificateur plus CAN de l'oscilloscope Yokogawa. La somme des deux fréquences est réalisée au moyen d'un additionneur à trois résistances, il est important de s'assurer au préalable, avec un analyseur de spectre analogique par exemple, que l'ensemble des deux générateurs et additionneur ne génère pas lui même des fréquences à $\left(2 F_{1}-F_{2}\right)$ et $\left(2 F_{2}-F_{1}\right)$.

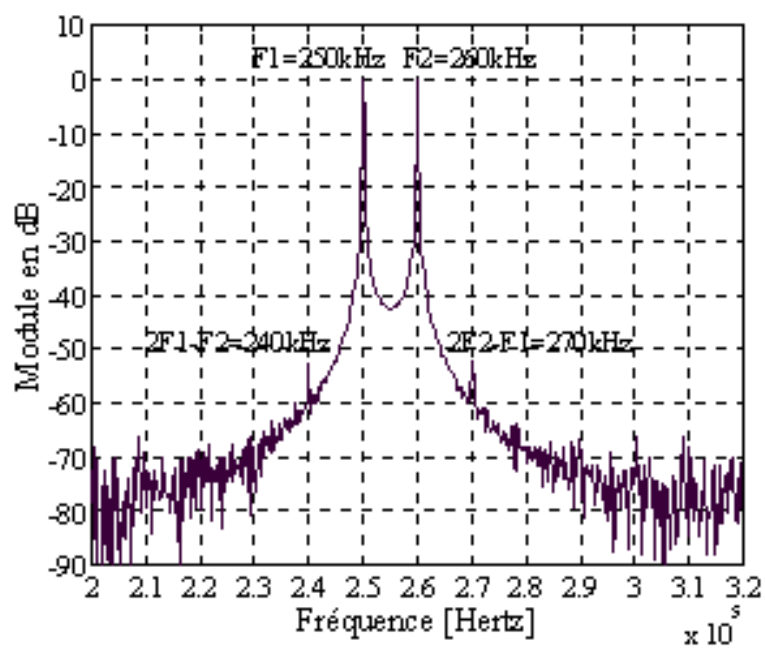

Fig. 6. Mise en évidence des non-linéarités de l'amplificateur et du CAN de l'oscilloscope numérique, le signal d'entrée est la somme de deux sinusoïdes ; résultat expérimental.

\subsection{Réponse en fréquence d'un système linéaire}

D'un point de vue théorique, la réponse en fréquence $H(f)$ d'un système linéaire est obtenue en calculant la transformée de Fourier de la réponse impulsionnelle $h(t): H(f)=T F[h(t)]$. En pratique, l'impulsion $e(t)$ appliquée au système a une largeur $\theta$ finie. Pour obtenir la réponse en fréquence du système à partir du calcul de la $F F T$, les échantillons $g\left(k T_{e}\right)$ de la réponse du système, différents des $h\left(k T_{e}\right)$, doivent être codés sur $N$ bits. Il est donc intéressant d'étudier : i) l'influence de la largeur $\theta$ et ii) les limitations de la FFT dans une telle analyse. L'objet de l'étude est un filtre du deuxième ordre (inductance $L$, résistance $R$ et capacité $C$ ) que les étudiants ont déjà rencontré soit en électronique soit encore en automatique. La fréquence de résonance $F_{0}$ et le coefficient de surtension $Q$ sont respectivement égaux à $2,8 \mathrm{kHz}$ et 3 . Plusieurs expériences sont proposées aux étudiants :

a) $\theta=1 \mu$ s et $F_{e}=2 \mathrm{MHz}$ : le module de la $F F T$ est donné à la figure 7. Jusqu'à environ $20 \mathrm{kHz}$, le résultat de la FFT est en très bon accord avec la courbe déduite d'un calcul analytique utilisant les impédances complexes. Pour illustrer la technique de remplissage mentionnée au $\S 2$, les $M=10^{4}$ points acquis sont complétés par $Q=\left(2^{14}-2^{10}\right)$

zéros, en conséquence le pas de calcul de la FFT est égal à

$$
\frac{F_{e}}{M+Q}=122 \mathrm{~Hz}
$$
d'échantillonnage élevée, l'absence de filtre antirepliement n'est pas ici un problème, en effet la limitation de la plage de fréquence intéressante est principalement due au bruit et plus particulièrement au bruit de quantification. 


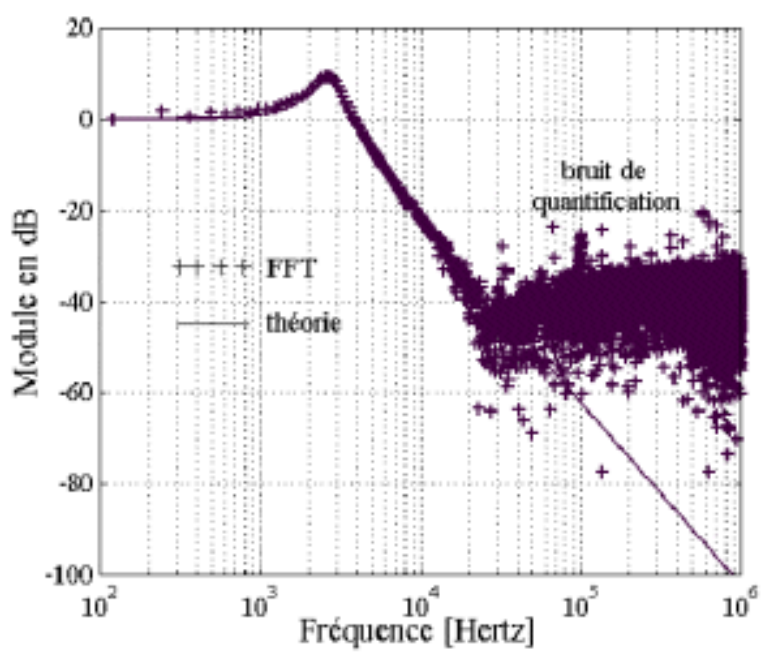

Fig. 7. FFT normalisée à $0 \mathrm{~dB}$ pour $f \rightarrow 0$ de la réponse à une impulsion de largeur $\theta=1 \mu$ s d'un filtre du second ordre.

$\beta) \theta=200 \mu$ s et $F_{e}=2 \mathrm{MHz}$ : le module de la FFT est donné à la figure 8. Le résultat montre clairement que $G(f)=H(f) E(f)$ où $G(f)$ et $E(f)$ sont respectivement les transformées de Fourier de $g(t)$ et $e(t)$. La transformée de Fourier $E(f)$ présente des minimums pour les fréquences $5 \mathrm{kHz}, 10 \mathrm{kHz}$, c'est-à-dire pour des valeurs multiples de $1 / \theta$. Les fréquences de $5 \mathrm{kHz}, 10 \mathrm{kHz}$ n'étant pas des multiples exacts de $F_{e} / M$, c'est la raison pour laquelle on a des minimums et non des zéros.

Ces deux expériences illustrent bien l'intérêt de la FFT pour l'étude des systèmes linéaires. Il est évidemment intéressant de comparer cette méthode avec la méthode harmonique classique, aucune méthode ne permet d'accéder à $H(f)$ aux fréquences élevées, compte tenu du rapport signal/bruit faible dans ce domaine.

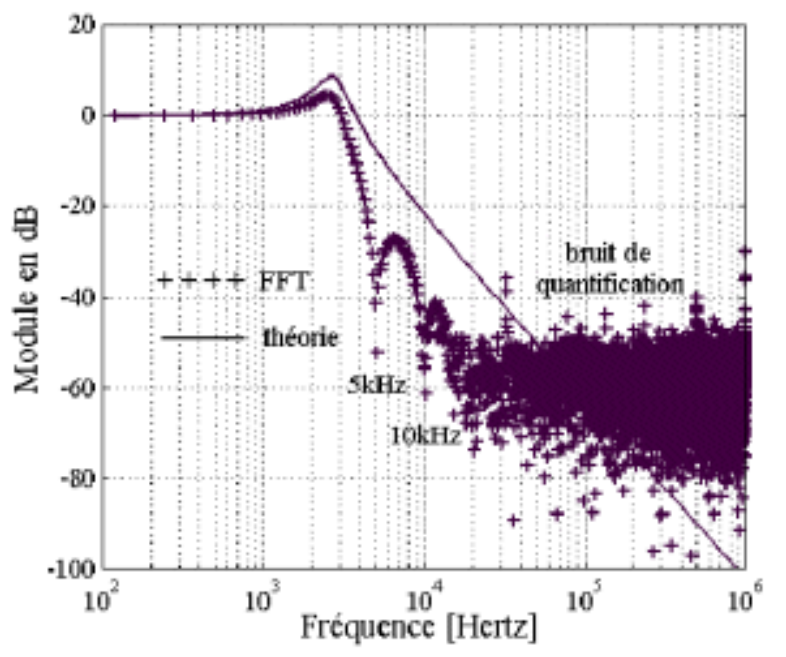

Fig. 8. FFT normalisée à $0 \mathrm{~dB}$ pour $f \rightarrow 0$ de la réponse à une impulsion de largeur $\theta=200 \mu$ s'un filtre du second ordre.

\section{Discussion sur la mesure du bruit de quantification}

Le bruit de quantification de la figure 5 , annoncé comme tel au § 3.2., est-il dû uniquement à la quantification sur $N$ bits des échantillons ? Quelle est l'importance du bruit physique qui est forcément présent ? Pour répondre à ces questions nous proposons de comparer les FFT obtenues expérimentalement avec le générateur et l'oscilloscope numérique aux FFT données par l'outil de simulation Simulink de Matlab. 
La figure 9 montre la FFT obtenue en simulation d'un signal sinusoïdal de fréquence $F_{I N}=50170,898 \mathrm{~Hz}$ échantillonné à $F_{e}=1 \mathrm{MHz}$ et codé avec 8 bits, pour se rapprocher de l'expérience nous avons additionné des harmoniques 2, 3 et 4 au fondamental. Il est évident que dans ce cas le bruit observé est uniquement dû au bruit de quantification, le bruit est a priori blanc sans raie apparente, excepté les raies dues aux harmoniques. On remarque cependant une dispersion des valeurs autour du plancher beaucoup plus importante que pour la FFT expérimentale. Pour faciliter la comparaison entre simulation et expérience nous avons reproduit la figure 5 à coté de la figure 9.

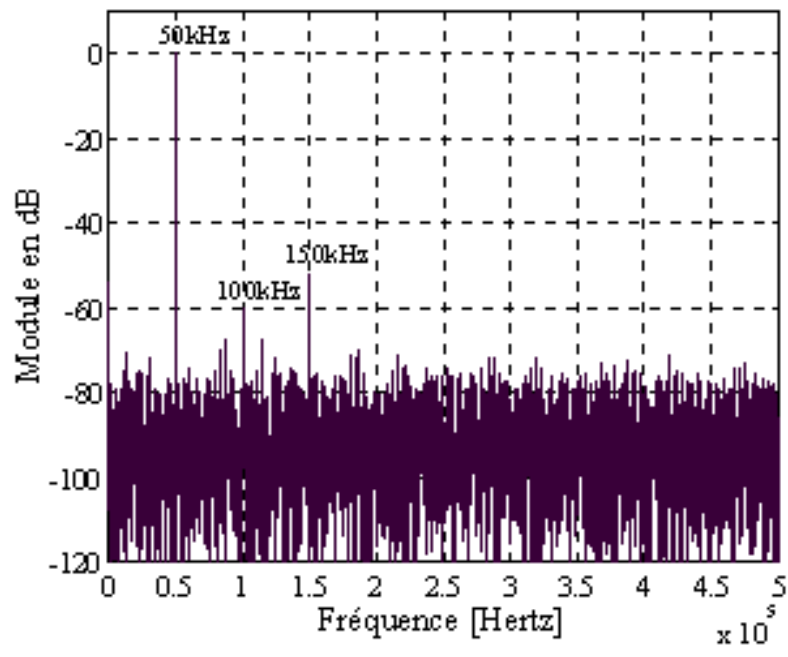

Fig. 9. FFT avec fenêtre rectangulaire, obtenue par simulation, d'un signal sinusoïdal de fréquence $F_{I N}=50170,898 \mathrm{~Hz}$, avec harmoniques 2,3 et 4 et codé sur 8 bits.

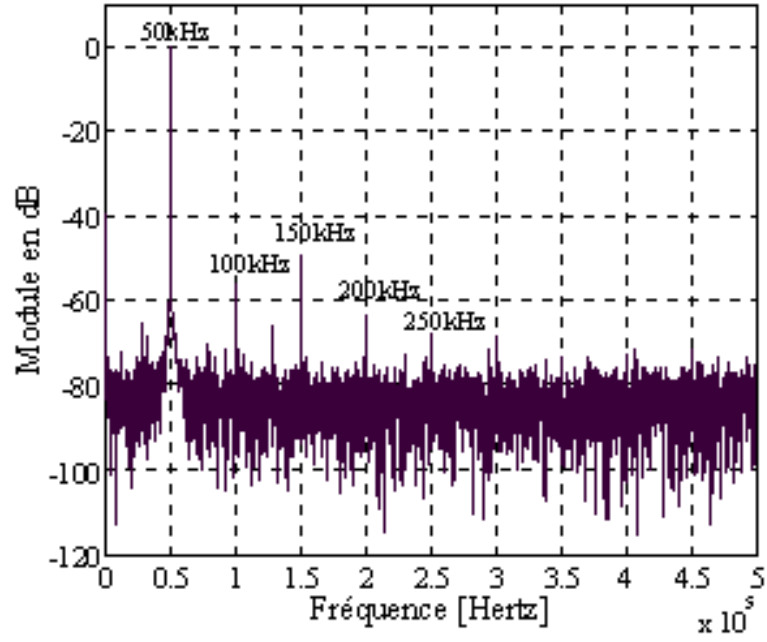

Fig. 5. FFT avec fenêtre rectangulaire d'un signal sinusoïdal de fréquence $F_{I N}=50170,898 \mathrm{~Hz}$ échantillonné à $1 \mathrm{MHz}$ et codé avec 8 bits; résultat expérimental.

Une simulation analogue réalisée avec une fréquence $F_{I N}=50 \mathrm{kHz}$ donne la FFT de la figure 10. Dans ce cas le bruit de quantification est très éloigné d'un bruit blanc, en effet il se présente sous formes de raies aux fréquences multiples de $50 \mathrm{kHz}$. Il s'agit d'un cas particulier de cohérence, en effet les échantillons quantifiés sont périodiques et de période égale à 20 microsecondes. On peut obtenir un résultat analogue avec des fréquences $F_{I N}$ telles que $\frac{F_{I N}}{F_{e}}=\frac{P}{R} \underset{\text { avec } P \text { et } R \text { entiers et premiers entre eux, et avec } R<M \text {. Pour } F_{I N} \text {, le rapport }}{\frac{P}{R}}=\frac{1}{20}$, la période des

échantillons quantifiés est égale à

$$
\frac{P}{F_{I N}}=20 \mu s
$$

, d'où les raies équidistantes de $50 \mathrm{kHz}$ sur la figure 10 . On pourra

$$
F_{I N}=50170,898 \mathrm{~Hz}=K \frac{F_{e}}{M}
$$

remarquer que dans le cas de la FFT de la figure 9 où $\quad$ avec $K=411, P=411$ et $R=8192$, c'est donc un cas limite car $M=8192$. On peut vérifier par exemple par simulation que pour $K=410$, $\frac{P}{\bar{R}}=\frac{205}{4096}$ et donc $R=4096<8192$, que le bruit de quantification présente des pics.

La figure 11 montre la FFT obtenue expérimentalement avec $F_{I N}=50 \mathrm{kHz}$ et $F_{e}=1 \mathrm{MHz}$, le bruit apparaît comme blanc. On remarque cependant des raies aux fréquences $350 \mathrm{kHz}, 400 \mathrm{kHz}$ et $450 \mathrm{kHz}$ qui sont la signature d'un reste de cohérence, ces raies sont absentes sur la FFT de la figure 5.

La différence essentielle entre les résultats expérimentaux et de simulation est le bruit physique provenant d'une part du générateur et d'autre part de l'amplificateur de l'oscilloscope. Le bruit physique a tendance à détruire la cohérence et donc à « blanchir » le spectre de raies. L'introduction de bruit pour «blanchir » le bruit de quantification est une technique quelques fois utilisée en conversion analogique numérique, elle porte le nom de dithering [10]. 


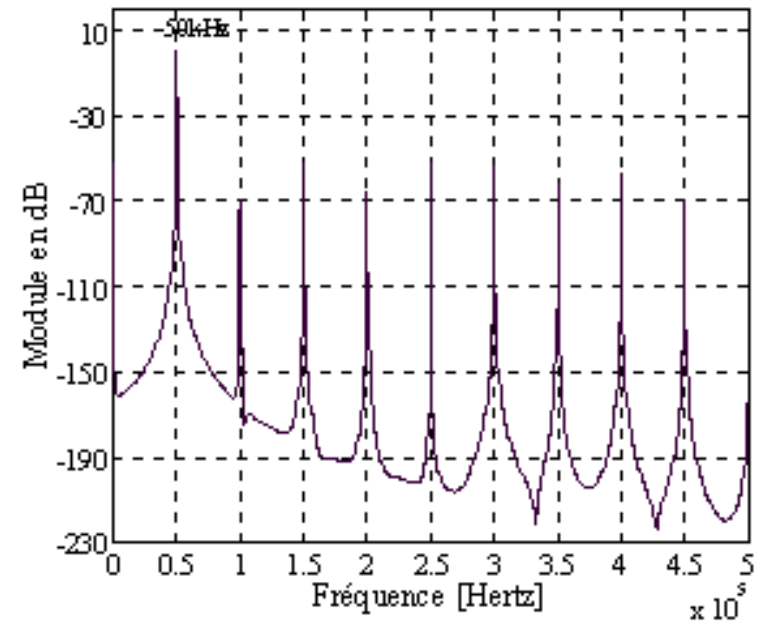

Fig. 10. FFT avec fenêtre de Hanning, obtenue par simulation, d'un signal sinusoïdal de fréquence $F_{I N}=50 \mathrm{kHz}$ avec harmoniques 2,3 et 4 et codé sur 8 bits.

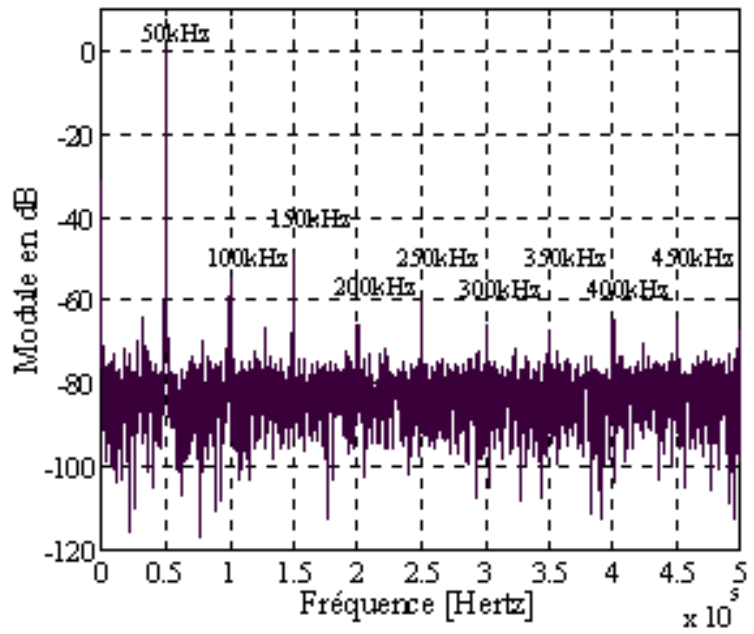

Fig.11. FFT avec fenêtre de Hanning d'un signal sinusoïdal de fréquence $F_{I N}=50 \mathrm{kHz}$ échantillonné à $1 \mathrm{MHz}$ et codé sous 8 bits ; résultat expérimental.

Compte tenu des différences entre les résultats expérimentaux (Fig. 5 et 11) et de simulation (Fig. 9 et 10), il faut donc admettre que les planchers de bruit des figures 5 et 11 sont perturbés par le bruit physique. On peut cependant raisonnablement s'attendre à une contribution faible du bruit physique vu que les valeurs mesurées des planchers de bruit sont proches de celles attendues.

Pour compléter l'étude nous avons donc effectuer des simulations en additionnant du bruit physique au signal comme le montre le schéma Simulink de la figure 12. Les points stockés dans la mémoire 1 sont utilisés pour calculer la FFT de la figure 10, dans ce cas particulier aucun bruit physique n'est additionné au signal. Les points stockés dans les mémoires 2 et 3 sont utilisés pour calculer les FFT des figures 13 et 14 suivantes.

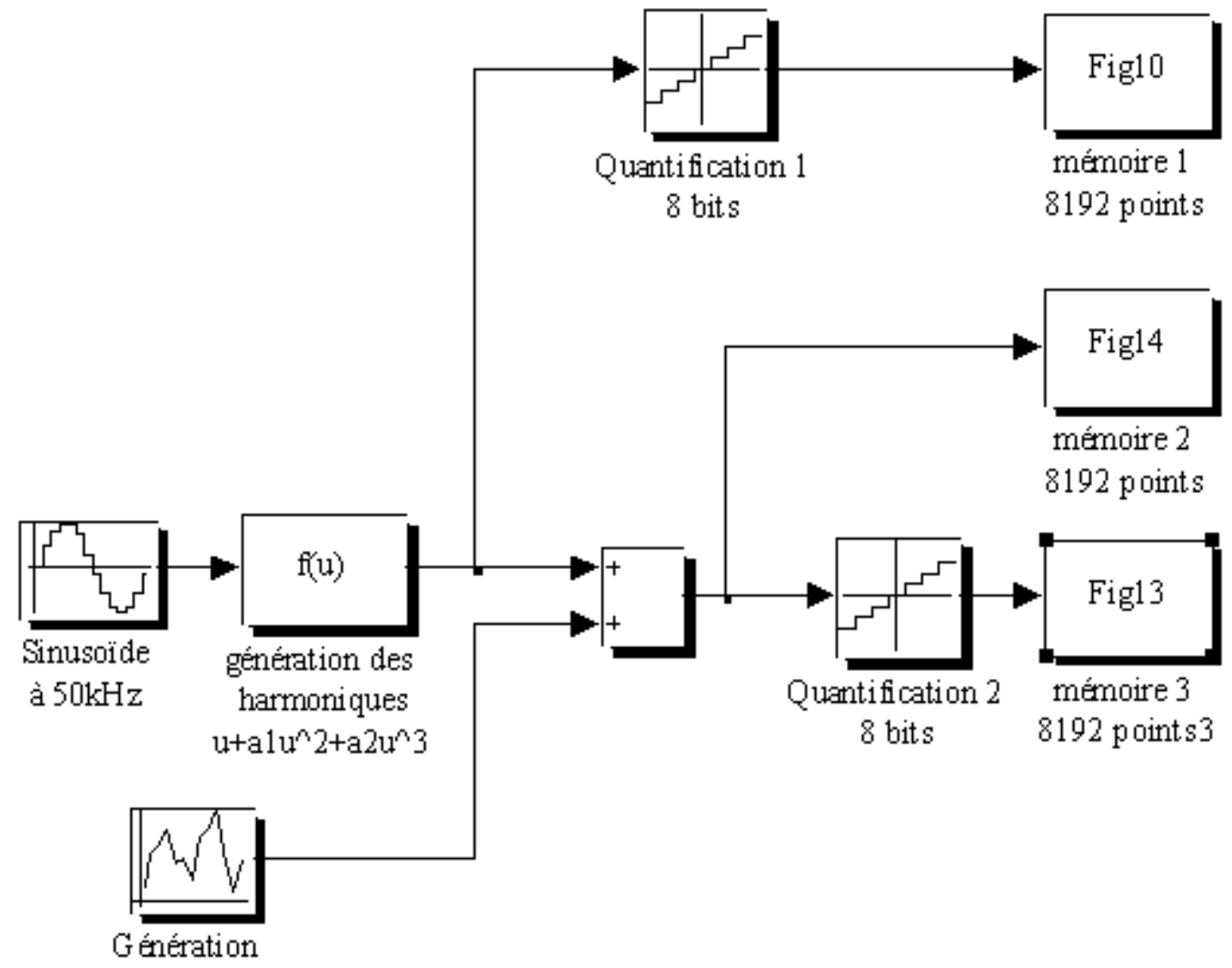

du bruit physique

Fig. 12. Schéma Simulink pour l'étude du bruit de quantification et du bruit physique. 
La FFT de la figure 13 est obtenue en additionnant un bruit physique au signal de fréquence $F_{I N}=50 \mathrm{kHz}$, les amplitudes des raies du bruit de quantification de la figure 10 ont diminué et le bruit est étalé en fréquence, c'est l'opération de dithering. La FFT de la figure 13 est très similaire à la FFT de la figure 11 obtenue expérimentalement. Le plancher de bruit est voisin des - $86 \mathrm{~dB}$ attendus car le bruit physique est à environ - $10 \mathrm{~dB}$ en dessous du plancher de bruit comme le montre la figure 14. En additionnant le même niveau de bruit physique au signal de fréquence $F_{I N}=50170,898 \mathrm{~Hz}$, on obtient la FFT de la figure 15 qui est à comparer à la FFT de la figure 9. Là encore le bruit physique détruit la cohérence et a tendance à blanchir le spectre pour donner une FFT similaire à celle obtenue expérimentalement et donnée en figure 5.

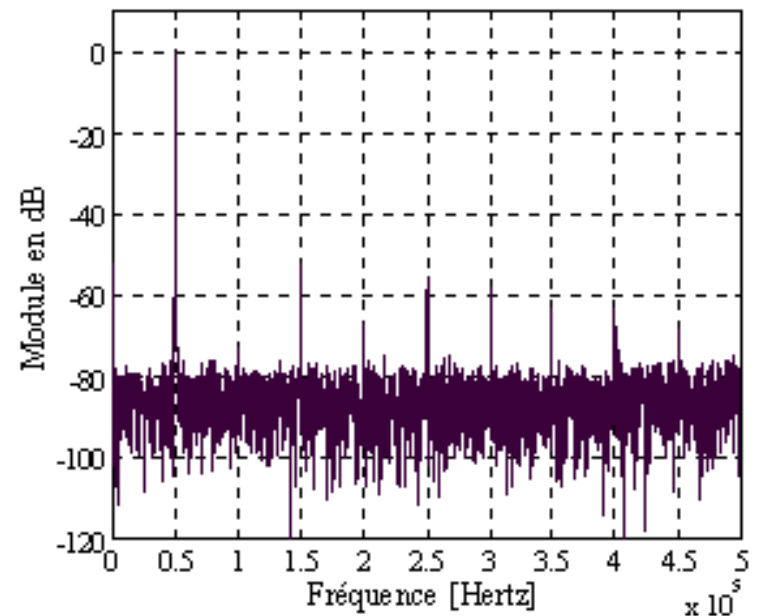

Fig. 13. FFT avec fenêtre de Hanning, obtenue par simulation, d'un signal sinusoïdal de fréquence $F_{I N}=50 \mathrm{kHz}$ avec harmoniques 2,3 et 4 et codé avec 8 bits. Un bruit physique est additionné au signal.

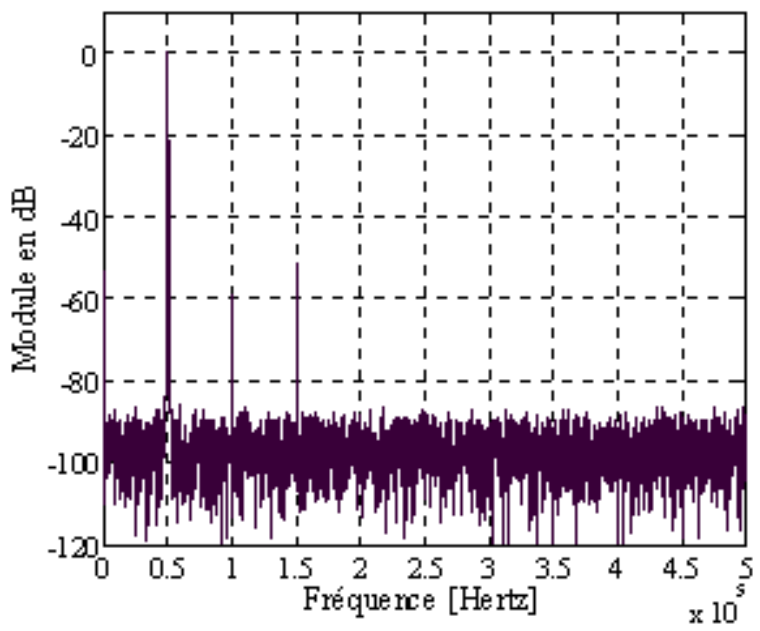

Fig. 14. FFT avec fenêtre de Hanning, obtenue par simulation, d'un signal sinusoïdal de fréquence $F_{I N}=50 \mathrm{kHz}$ avec harmoniques 2,3 et 4 et bruit. Les échantillons ne sont pas quantifiés. Le bruit physique est à environ - $10 \mathrm{~dB}$ sous le plancher de bruit de la figure 13.

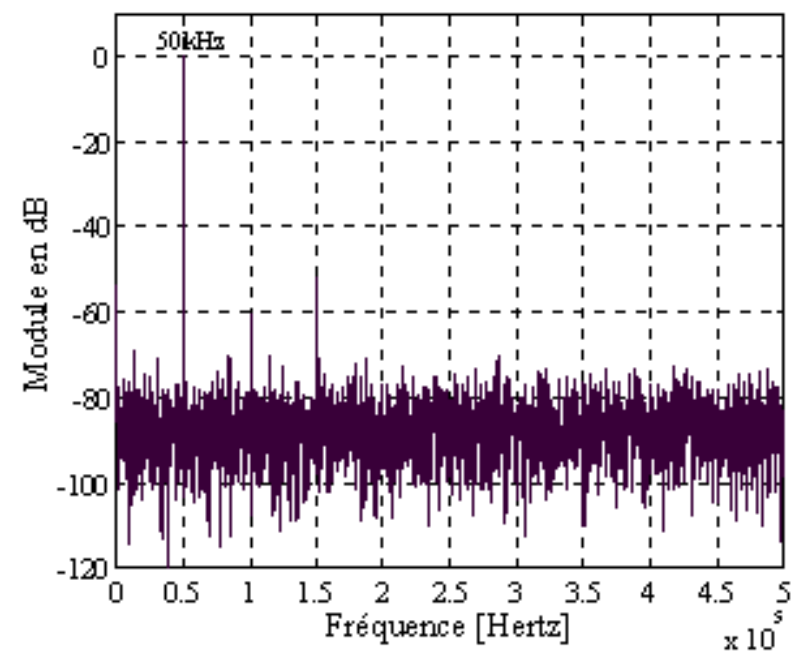

Fig. 15. FFT avec fenêtre rectangulaire, obtenue par simulation, d'un signal sinusoïdal de fréquence $F_{I N}=50170,898 \mathrm{~Hz}$ avec harmoniques 2,3 et 4 et codé sur 8 bits. Un bruit physique est additionné au signal. Cette FFT est similaire à celle de la figure 5 obtenue expérimentalement. 


\section{Conclusion}

Les outils de traitement des signaux comme la FFT facilitent grandement la tâche des techniciens, ingénieurs et chercheurs travaillant dans de nombreux secteurs et pas seulement ceux de l'EEA. D'un point de vue pédagogique, ces outils, facilement accessibles, ont cependant tendance à compliquer quelque peu la tâche des enseignants, surtout des enseignants intervenant dans les cycles techniques courts où le recours aux mathématiques est limité. Faut-il alors renoncer à utiliser ces outils sous prétexte qu'ils sont trop compliqués ? La réponse est évidemment non, en conséquence il nous faut développer une pédagogie adaptée pour démystifier ceux-ci.

C'est dans cet état d'esprit que nous avons préparé des expériences utilisant la FFT. Ces expériences ont un double objectif, le premier est de présenter l'outil proprement dit, le second est d'appliquer l'outil à l'étude de systèmes en rapport avec le contenu de la formation.

S'agissant du premier objectif, nous montrons que par des choix convenables de signaux il est relativement aisé d'introduire les notions de résolution et de dynamique et par conséquent d'aborder le choix d'une fenêtre de pondération.

Pour ce qui est des applications de la FFT, nous avons choisi d'illustrer son intérêt en étudiant partiellement un CAN, c'est ainsi que nous revisitons la notion de bruit de quantification. Nous proposons également une expérience pour identifier les non-linéarités de l'amplificateur et du CAN d'un oscilloscope numérique. La dernière expérience concerne le relevé de la réponse en fréquence d'un filtre du deuxième ordre. La FFT permet, sous certaines conditions, d'accéder rapidement à $H(f)$.

Les comparaisons entre simulations et expériences du $\S 4$ permettent d'approfondir le rôle du bruit physique dans la mesure du bruit de quantification. Cette dernière partie ne peut pas être abordée au niveau bac +2 , mais comme nous le montrons au $\S 3$, il n'est pas nécessaire dans une première approche de tenir compte du bruit physique, tout au moins avec les conditions expérimentales retenues.

\section{Références bibliographiques}

[1] Bulletin Officiel du Ministère de l'Éducation nationale et du Ministère de la Recherche N³4 du 20/09/2001, Organisation des études conduisant au DUT spécialité "génie électrique et informatique industrielle" : http://www.education.gouv.fr/bo/2001/34/sup.htm (consulté le 9 mai 2003).

[2] C. Gasquet et P. Witomski, Analyse de Fourier et Applications (Masson, 1995).

[3] M. Bellanger, Traitement numérique du signal, Théorie et pratique, $7^{\mathrm{ème}}$ éd. (Dunod, 2002).

[4] G. Blanchet et J. Prado, Méthodes numériques pour le traitement du signal (Masson, 1991).

[2] F. Cottet, Traitement des signaux et acquisition de données (Dunod, 1997).

[6] F.J. Harris, On the use of windows for harmonic analysis with Discrete Fourier Transform, Proc. IEEE 66

(1978) 51.

[7] Site WEB Agilent - Test \& Measurement :

http://www.agilent.com (consulté le 9 mai 2003).

résolution $10 \mu \mathrm{Hz}$, précision $90 \mathrm{ppm} / 90$ jours, distorsion harmonique : de $20 \mathrm{kHz}$ à $100 \mathrm{kHz}:-60 \mathrm{dBc}$, de $100 \mathrm{kHz}$ à $1 \mathrm{MHz}$ : $-45 \mathrm{dBc}$, signaux parasites non harmoniques $<-65 \mathrm{dBc}$ du continu à $1 \mathrm{MHz}$, bruit de phase : $<-55 \mathrm{dBc}$ dans une bande de $30 \mathrm{kHz}$.

[8] Site WEB Yokogawa : http://www.yokogawa.com (consulté le 9 mai 2003).

[9] L. Gaddy, Selecting an A/D converter, Burr-Brown Application Bulletin AB-098 :

http://www-s.ti.com/sc/psheets/sbaa004/sbaa004.pdf (consulté le 9 mai 2003).

[10] J. Laroche, Traitement des signaux Audio-Fréquences (TELECOM Paris, 1995).

NB : les codes sources Matlab sont disponibles auprès des auteurs sur demande. 